\title{
A NOTE ON THE NET PREMIUM FOR A GENERALIZED LARGEST CLAIMS REINSURANCE COVER
}

\author{
By Raoul M. Berglund \\ Department of Mathematics \\ Abo Akademi University
}

\begin{abstract}
In the present paper the author gives net premium formulae for a generalized largest claims reinsurance cover. If the claim sizes are mutually independent and identically 3-parametric Pareto distributed and the number of claims has a Poisson, binomial or negative binomial distribution, formulae are given from which numerical values can easily be obtained. The results are based on identities for compounded order statistics.
\end{abstract}

\section{KEYWORDS}

Net premium; Reinsurance; LCR; ECOMOR; Compounded order statistics.

\section{INTRODUCTION}

An expression for the pure premium for the largest claim reinsurance cover was already introduced by AMMETER (1964a) and for the $p$ largest claims reinsurance cover by AMMETER (1964b). Simple formulae were presented under the assumptions that the claim sizes obeyed a one parametric Pareto distribution and the number of claims was Poisson distributed. For the same claim size distribution KUPPER (1971) gave a formula for the largest claim reinsurance when the number of claims was geometrically distributed and Ciminelli (1976) considered a negative binomial distribution. BERLINGER (1972) extended the results by AMMETER and deduced the variance for the $p$ largest claims reinsurance cover. Net premium for a general claim size and claim number distribution was given by KREMER (1985) and for some generalized claim number distributions and a general claim size distribution by KREMER (1988a). The results in the latter were, however, not so practical for a specific claim size distribution. The author of this paper gives net premium formulae for a generalized largest claims reinsurance cover, assuming that the claim sizes are mutually independent and identically 3-parametric Pareto distributed and when the number of claims has a Poisson, binomial or negative binomial distribution. The formulae presented in this paper are simple and easily calculated. 


\section{PRELIMINARIES}

From now on, let $X_{1}, X_{2}, \ldots, X_{N}$ denote non-negative, mutually independent and identically distributed claim sizes, which are independent of the number of claims $N$ that occur in a given time period. Denote by

$$
X_{N: 1} \geq X_{N: 2} \geq \ldots \geq X_{N: N}
$$

the claims ordered in a decreasing size. The $i$-th largest claim is called the $i$-th ordered claim or more generally the $i$-th compounded order statistic. Let

$$
f_{i}:[0, \infty) \rightarrow[0, \infty)
$$

$(i \geq l)$ be measurable functions, that satisfy

$$
f_{i}(0)=0 \text { and } \sum_{i=1}^{n} f_{i}\left(y_{i}\right) \in\left[0, \sum_{i=1}^{n} y_{i}\right]
$$

for all $0 \leq y_{n} \leq \ldots \leq y_{2} \leq y_{1}$. This representation was first made by KREMER (1982) and the following main definition by KREMER (1984):

Definition. The reinsurance treaty defined by

$$
R_{N}\left(X_{N: 1}, X_{N: 2}, \ldots, X_{N: N}\right)=R_{N}=\sum_{i=1}^{N} f_{i}\left(X_{N: i}\right)
$$
which determins the reinsurers share of the total $\operatorname{loss} \sum_{i=1}^{N} X_{i}$, is called a reinsurance
treaty based on ordered claims.

We are especially interested in the case

$$
f_{i}(x)=a_{i} x
$$

where $a_{i}, i \geq 1$, are real constants. This reinsurance treaty is defined as the generalized largest claims cover (KREMER 1988b). We get for

$$
a_{1}=a_{2}=\ldots=a_{p}=1, a_{i}=0 \forall i>p
$$

the so called $\operatorname{LCR}(p)$ treaty covering the $p$ largest claims and for

$$
a_{1}=a_{2}=\ldots=a_{p-1}=1, a_{p}=I-p, a_{i}=0 \forall i>p
$$

the so called $\operatorname{ECOMOR}(p)$ treaty covering all claims in excess of the $p$-th largest claim.

We will subsequently use some special functions. The incomplete gamma function is defined as

$$
\Gamma(a ; x)=\int_{0}^{x} e^{-u} u^{a-1} d u \quad, a>0, x \geq 0
$$


and the complete gamma function as $\lim _{x \rightarrow \infty} \Gamma(a ; x)=\Gamma(a)$. The incomplete beta function is defined as

$$
B(a ; b ; x)=\int_{0}^{x} u^{a-1}(1-u)^{b-1} d u=\int_{0}^{\frac{x}{1-x}} u^{a-1}(1+u)^{-(a+b)} d u \quad, a, b>0,0 \leq x<1
$$

and the complete beta function as $\lim _{x \rightarrow l} B(a ; b ; x)=B(a ; b)$. The complete beta function and the complete gamma function are related by

$$
B(a ; b)=\frac{\Gamma(a) \Gamma(b)}{\Gamma(a+b)}
$$

\section{FORMULAE FOR THE NET PREMIUM}

The two most common risk loaded premium principles, the variance principle and the standard deviation principle, are based on the expectation and the variance of a certain risk. For a generalized largest claims reinsurance cover the expectation is given by

$$
E\left[R_{N}\right]=\sum_{i=l}^{\infty} a_{i} E\left[X_{N: i}\right]
$$

and the variance by

$$
\operatorname{Var}\left[R_{N}\right]=\sum_{i=1}^{\infty} a_{i}^{2} E\left[X_{N: i}^{2}\right]+2 \sum_{j=2}^{\infty} \sum_{i=1}^{j-1} a_{i} a_{j} E\left[X_{N: i} X_{N: j}\right]-\left(\sum_{i=1}^{\infty} a_{i} E\left[X_{N: i}\right]\right)^{2} .
$$

The following theorem is due to Ciminelli (1976) and Kremer (1985), where

$$
\phi(s)=\sum_{n=0}^{\infty} P(N=n) s^{n}
$$

denotes the probability generating function of $N$, which is assumed to have derivatives $\phi^{(i)}$ on $(0,1)$ of each order $i \geq 1$.

Theorem 1. If the claim sizes $X_{1}, X_{2}, \ldots, X_{N}$ have a continuous distribution function $F$ the density function of the $i$-th ordered claim is given by

$$
P\left(X_{N: i}=x\right)=\frac{1}{\Gamma(i)} f(x)[l-F(x)]^{i-1} \phi^{(i)}(F(x))
$$

and the joint density function of the $i$-th and $j$-th ordered claims $(0<i<j)$ is given by

$P\left(X_{N: i}=x_{i}, X_{N: j}=x_{j}\right)=\frac{l}{\Gamma(i) \Gamma(j-l)}\left[I-F\left(x_{i}\right)\right]^{i-1}\left[F\left(x_{i}\right)-F\left(x_{j}\right)\right]^{j-i-1} \phi^{(j)}\left(F\left(x_{j}\right)\right) f\left(x_{i}\right) f\left(x_{j}\right)$. 
Theorem 2. If the claim sizes $X_{1}, X_{2}, \ldots, X_{N}$ have a continuous distribution function $F$ the $k$-th moment around the origin of the $i$-th ordered claim is given by

$$
E\left[X_{N: i}^{k}\right]=\frac{1}{\Gamma(i)} \int_{0}^{l} F^{-l}(u)^{k}[1-u]^{i-1} \phi^{(i)}(u) d u
$$

and the expectation of the cross product of the $i$-th and $j$-th ordered claims $(0<i<j)$ is given by

$$
\begin{gathered}
E\left[X_{N: i} X_{N: j}\right]= \\
\frac{1}{\Gamma(i) \Gamma(j-i)} \int_{0}^{l} F^{-1}(v)(1-v)^{j-1} \phi^{(j)}(v) \int_{0}^{l} F^{-l}(1-u(1-v)) u^{i-1}(I-u)^{j-i-1} d u d v .
\end{gathered}
$$

Proof: The first part of the statement follows from theorem 1 after the substitution $u=F(x)$. For the second part we have for $0<i<j$ and $0 \leq X_{N: j} \leq X_{N: i}$ that

$$
\begin{gathered}
E\left[X_{N: i} X_{N: j}\right]= \\
C \int_{0}^{\infty} \int_{x_{j}}^{\infty} x_{i} x_{j}\left[1-F\left(x_{i}\right)\right]^{i-I}\left[F\left(x_{i}\right)-F\left(x_{j}\right)\right]^{j-i-l} \phi^{(j)}\left(F\left(x_{j}\right)\right) f\left(x_{i}\right) f\left(x_{j}\right) d x_{i} d x_{j},
\end{gathered}
$$

where

$$
C=\frac{1}{\Gamma(i) \Gamma(j-i)}
$$

After substituting $u=\frac{I-F\left(x_{i}\right)}{I-F\left(x_{j}\right)}$ and $v=F\left(x_{j}\right)$ we obtain

$$
\begin{gathered}
E\left[X_{N: i} X_{N: j}\right]= \\
C \int_{0}^{I} \int_{0}^{l} F^{-I}(I-u(I-v)) F^{-1}(v) u^{i-1}[I-v]^{j-1}[I-u]^{j-i-1} \phi^{(j)}(v) d u d v= \\
C \int_{0}^{I} F^{-1}(v)[I-v]^{j-1} \phi^{(j)}(v) \int_{0}^{I} F^{-1}(I-u(I-v)) u^{i-I}[I-u]^{j-i-l} d u d v .
\end{gathered}
$$

From now on we will focus on the case where the claim sizes are distributed according to the 3-parametric Pareto distribution

$$
F(x)=1-\left(\frac{d+\beta}{x+\beta}\right)^{\alpha} \quad x \geq d>0,
$$


where the parameters $\alpha, \beta$ and $d$ satisfy $\alpha>0$ and $b>-d$. The distribution (3.1) is the most used claim size distribution, especially if there is a possibility of large claims. In the literature the 3-parametric Pareto distribution is sometimes also called the "shifted" Pareto distribution (RYTGAARD 1990) or the complete Pareto distribution (DAYKIN et al. 1994). Since

$$
F^{-1}(x)=\frac{d+\beta}{(1-x)^{\frac{1}{\alpha}}}-\beta
$$

the expectations of theorem 2 becomes after binomial expansion and simplifications

$$
E\left[X_{N: i}^{k}\right]=\frac{1}{\Gamma(i)} \sum_{h=0}^{k}\left(\begin{array}{l}
k \\
h
\end{array}\right)(d+\beta)^{k-h}(-\beta)^{h} \int_{0}^{l}(1-u)^{i-\frac{k-h}{c k}-1} \phi^{(i)}(u) d u
$$

and for $\alpha>\frac{1}{i}$

$$
\begin{gathered}
E\left[X_{N: i} X_{N: j}\right]=\frac{1}{\Gamma(i)} \\
{\left[A_{1} \int_{0}^{l}(I-v)^{j-\frac{2}{{ }^{\prime}}-1} \phi^{(j)}(v) d v-A_{2} \int_{0}^{l}(I-v)^{j-\frac{1}{n}-1} \phi^{(j)}(v) d v+A_{3} \int_{0}^{l}(I-v)^{j-1} \phi^{(j)}(v) d v\right]}
\end{gathered}
$$

where

$$
\begin{aligned}
& A_{1}=(d+\beta)^{2} \frac{\Gamma\left(i-\frac{1}{\alpha}\right)}{\Gamma\left(j-\frac{I}{\alpha}\right)} \\
& A_{2}=\beta(d+\beta)\left[\frac{\Gamma(i)}{\Gamma(j)}+\frac{\Gamma\left(i-\frac{1}{\alpha}\right)}{\Gamma\left(j-\frac{1}{\alpha}\right)}\right] \\
& A_{3}=\beta^{2} \frac{\Gamma(i)}{\Gamma(j)}
\end{aligned}
$$

The restriction on the parameter $\alpha$ is needed to get a finite expression. Assuming further that the number of claims $N$ is Poisson distributed

$$
P(N=n)=\frac{\lambda^{n}}{n !} e^{-\lambda} \quad \lambda>0, n \geq 0
$$

negative binomially distributed

$$
P(N=n)=\frac{\Gamma(r+n)}{\Gamma(r) n !}\left(\frac{1}{I+\lambda}\right)^{r}\left(\frac{\lambda}{I+\lambda}\right)^{n} \quad r, \lambda>0, n \geq 0
$$


or binomially distributed

$$
P(N=n)=\left(\begin{array}{c}
m \\
n
\end{array}\right) q^{n}(1-q)^{m-n} \quad 0 \leq q \leq 1, n=0, l, \ldots, m,
$$

where $m$ is a non-negative integer, we have the following corollaries.

Corollary 3. Assume that the claim sizes $X_{1}, X_{2}, \ldots, X_{N}$ are Pareto distributed (3.1) and that the claim number $N$ is Poisson distributed (3.2). Then the $k$-th moment around the origin of the $i$-th ordered claim is, for $\alpha>\frac{k}{i}$ given by

$$
E\left[X_{N: i}^{k}\right]=\frac{1}{\Gamma(i)} \sum_{h=0}^{k}\left(\begin{array}{l}
k \\
h
\end{array}\right)(d+\beta)^{k-h}(-\beta)^{h} \lambda^{\frac{k-h}{\kappa}} \Gamma\left(i-\frac{k-h}{\alpha} ; \lambda\right)
$$

and the expectation of the cross product of the $i$-th and $j$-th ordered claims $(0<i<j)$ is, for $\alpha>\max \left\{\frac{l}{i}, \frac{2}{j}\right\}$, given by

$$
E\left[X_{N: i} X_{N: j}\right]=\frac{1}{\Gamma(i)}\left[A_{1} \lambda^{\frac{2}{\alpha}} \Gamma\left(j-\frac{2}{\alpha} ; \lambda\right)-A_{2} \lambda^{\frac{l}{\Gamma}} \Gamma\left(j-\frac{1}{\alpha} ; \lambda\right)+A_{3} \Gamma(j ; \lambda)\right] .
$$

Proof: Since the $j$-th derivative of the probability generating function $\phi$ for a Poisson distributed random variable (3.2) is given by

$$
\phi^{(j)}(s)=\lambda^{j} e^{\lambda(s-I)}
$$

we have, for $\gamma>0$, that

$$
\int_{0}^{l}(1-u)^{\gamma-1} \phi^{(j)}(u) d u=\lambda^{j} \int_{0}^{l}(1-u)^{\gamma-1} e^{\lambda(u-l)} d u .
$$

After the substitution $t=\lambda(l-u)$ we obtain

$$
\begin{aligned}
\int_{0}^{1}(1-u)^{\gamma-1} \phi^{(j)}(u) d u & =\lambda^{j-\gamma} \int_{0}^{\lambda} t^{\gamma-1} e^{-t} d t \\
& =\lambda^{j-\gamma} \Gamma(\gamma ; \lambda),
\end{aligned}
$$

which gives the result.

Corollary 4. Assume that the claim sizes $X_{I}, X_{2} \ldots, X_{N}$ are Pareto distributed (3.1) and that the claim number $N$ is negative binomially distributed (3.3). Then the $k$-th moment around the origin of the $i$-th ordered claim is, for $\alpha>\frac{k}{i}$, given by 


$$
E\left[X_{N: i}^{k}\right]=\frac{1}{B(i ; r)} \sum_{h=0}^{k}\left(\begin{array}{l}
k \\
h
\end{array}\right)(d+\beta)^{k-h}(-\beta)^{h} \lambda^{\frac{k-h}{\alpha}} B\left(i-\frac{k-h}{\alpha} ; r+\frac{k-h}{\alpha} ; \frac{\lambda}{I+\lambda}\right)
$$

and the expectation of the cross product of the $i$-th and $j$-th ordered claims $(0<i<j)$ is, for $\alpha>\max \left\{\frac{1}{i}, \frac{2}{j}\right\}$, given by

$$
\begin{gathered}
E\left[X_{N: i} X_{N: j}\right]=\frac{1}{B(j ; r)} \frac{\Gamma(j)}{\Gamma(i)} \\
{\left[A_{1} \lambda^{\frac{2}{\underline{n}}} B\left(j-\frac{2}{\alpha} ; r+\frac{2}{\alpha} ; \frac{\lambda}{1+\lambda}\right)-A_{2} \lambda^{\frac{1}{\alpha}} B\left(j-\frac{1}{\alpha} ; r+\frac{1}{\alpha} ; \frac{\lambda}{1+\lambda}\right)+A_{3} B\left(j ; r ; \frac{\lambda}{1+\lambda}\right)\right] .}
\end{gathered}
$$

Proof: Since the $j$-th derivative of the probability generating function $\phi$ for a negative binomially distributed random variable (3.3) is given by

$$
\phi^{(j)}(s)=\frac{\Gamma(r+j)}{\Gamma(r)} \lambda^{j}[I-\lambda(s-l)]^{-(r+j)}
$$

we have, for $\gamma>0$, that

$$
\int_{0}^{l}(I-u)^{\gamma-l} \phi^{(j)}(u) d u=\frac{\Gamma(r+j)}{\Gamma(r)} \lambda^{j} \int_{0}^{l}(1-u)^{\gamma-1}[1-\lambda(u-l)]^{-(r+j)} d u .
$$

After the substitution $t=\lambda(I-u)$ we obtain

$$
\begin{aligned}
\int_{0}^{l}(1-u)^{\gamma-1} \phi^{(j)}(u) d u & =\frac{\Gamma(r+j)}{\Gamma(r)} \lambda^{j-\gamma} \int_{0}^{\lambda} t^{\gamma-1}(1+t)^{-(r+j)} d t \\
& =\frac{\Gamma(r+j)}{\Gamma(r)} \lambda^{j-\gamma} B\left(\gamma ; r+j-\gamma ; \frac{\lambda}{1+\lambda}\right),
\end{aligned}
$$

from which the result follows after simplification.

Corollary 5. Assume that the claim sizes $X_{1}, X_{2} \ldots, X_{N}$ are Pareto distributed (3.1) and that the claim number $N$ is binomially distributed (3.4). Then the $k$-th moment around the origin of the $i$-th ordered claim is, for $\alpha>\frac{k}{i}$, given by

$$
E\left[X_{N: i}^{k}\right]=i\left(\begin{array}{c}
m \\
i
\end{array}\right) \sum_{h=0}^{k}\left(\begin{array}{l}
k \\
h
\end{array}\right)(d+\beta)^{k-h}(-\beta)^{h} q^{\frac{k-h}{\alpha}} B\left(i-\frac{k-h}{\alpha} ; m-i+1 ; q\right)
$$

and the expectation of the cross product of the $i$-th and $j$-th ordered claims $(0<i<j)$ is, for $\alpha>\max \left\{\frac{1}{i}, \frac{2}{j}\right\}$, given by

$$
\begin{aligned}
E\left[X_{N: i} X_{N: j}\right] & =\left(\begin{array}{c}
m \\
j
\end{array}\right) \frac{\Gamma(j+1)}{\Gamma(i)}\left[A_{1} q^{\frac{2}{\alpha}} B\left(j-\frac{2}{\alpha} ; m-j+1 ; q\right)-A_{2} q^{\frac{1}{\alpha}} B\left(j-\frac{1}{\alpha} ; m-j+1 ; q\right)\right. \\
& \left.+A_{3} B(j ; m-j+1 ; q)\right]
\end{aligned}
$$


Proof: Since the $j$-th derivative of the probability generating function $\phi$ for a binomially distributed random variable (3.4) is given by

$$
\phi^{(j)}(s)=\frac{\Gamma(m+1)}{\Gamma(m-j+1)} q^{j}[q s+1-q]^{m-j} \quad j \leq m,
$$

we have, for $\gamma>0$, that

$$
\int_{0}^{l}(1-u)^{\gamma-1} \phi^{(j)}(u) d u=\frac{\Gamma(m+1)}{\Gamma(m-j+1)} q^{j} \int_{0}^{l}(1-u)^{\gamma-1}(q u+1-q)^{m-j} d u .
$$

After the substitution $t=q(1-u)$ we obtain

$$
\begin{aligned}
\int_{0}^{l}(I-u)^{\gamma-l} \phi^{(j)}(u) d u & =\frac{\Gamma(m+l)}{\Gamma(m-j+l)} q^{j-\gamma} \int_{0}^{q} t^{\gamma-l}(I-t)^{m-j} d t \\
& =\frac{\Gamma(m+l)}{\Gamma(m-j+l)} q^{j-\gamma} B(\gamma ; m-j+I ; q),
\end{aligned}
$$

from which the result follows after simplification.

If $0<\alpha<1$, which indicates a very heavy tailed distribution, we have according to the results above that the first moment around origin of a certain number of the largest ordered claims does not exist. We could therefore consider the number of ordered claims, for which the first moment around the origin does not exist, as a measure for how dangerous a Pareto distribution is. Since many computer programs have built-in routines for computing the complete gamma, incomplete gamma and the incomplete beta function, the expectations in results above can be calculated easily.

If the claim sizes obey an exponential distribution

$$
F(x)=1-e^{-\beta(x-a)} \quad \beta>0, x \geq a,
$$

we cannot get useful expressions for the moments around the origin and the cross product by applying theorem 2 . Using well known results from order statistics for a deterministic number of claims (DAVID 1970) and then the iterativity of the expectation operator, expression for the pure premium can be constructed. Exponentially distributed claim sizes have been studied by KUPPER (1971) and KREMER (1985 and 1986).

\section{A NUMERICAL EXAMPLE}

Let the distribution for the claim sizes be Pareto distributed (3.1) with $d=0$. For the insurance line under consideration the method of moments gives the following parameter estimates: $\hat{\alpha}=2.3401$ and $\hat{\beta}=13692$. Since the most import claim number distributions are the Poisson and the negative binomial, we will restrict 
the example to them. Using the same estimation method we have the following parameter estimates: Poisson $\hat{\lambda}=79.667$, negative binomial $\hat{\lambda}=1.0865$ and $\hat{r}=73.326$. We have the following numerical results:

Expectation of $\operatorname{LCR}(p)$ and $\operatorname{ECOMOR}(p)$ treaties

\begin{tabular}{|c|c|c|c|c|c|}
\hline$\underline{p}$ & Poisson & negative binomial & $p$ & Poisson & negative binomial \\
\hline 1 & 124597 & 124368 & 1 & 0 & 0 \\
\hline 2 & 190099 & 189738 & 2 & 59095 & 58997 \\
\hline 3 & 238679 & 238215 & 3 & 92937 & 92783 \\
\hline 4 & 278390 & 277837 & 4 & 119548 & 119350 \\
\hline 5 & 312395 & 311763 & 5 & 142369 & 142133 \\
\hline \multicolumn{3}{|c|}{$\operatorname{LCR}(p)$-treaty } & \multicolumn{3}{|c|}{$\operatorname{ECOMOR}(p)$-treaty } \\
\hline
\end{tabular}

Standard deviation of $\operatorname{LCR}(p)$ and $\operatorname{ECOMOR}(p)$ treaties

\begin{tabular}{|c|c|c|c|c|c|}
\hline$p$ & Poisson & negative binomial & $p$ & Poisson & negative binomial \\
\hline 1 & 178069 & $178 \quad 129$ & 1 & 0 & 0 \\
\hline 2 & 191632 & 191860 & 2 & 134587 & 134549 \\
\hline 3 & 198847 & 199254 & 3 & 182222 & 182206 \\
\hline 4 & 203797 & 204389 & 4 & 188799 & 188815 \\
\hline 5 & 207581 & 208363 & 5 & 193255 & 193405 \\
\hline \multicolumn{3}{|c|}{ LCR $(p)$-treaty } & \multicolumn{3}{|c|}{ ECOMOR $(p)$-treaty } \\
\hline
\end{tabular}

The difference between the numerical values for Poisson and the negative binomial cases is quite small. If we assume that in the incomplete beta function $b$ is large and $a$ is bounded we have the following asymptotic representation (ABRAMOWITZ and STEGUN 1972)

$$
\frac{B(a ; b ; x)}{B(a ; b)}=\frac{\Gamma\left(a ; \frac{x(2 b+a-1)}{2-x}\right)}{\Gamma(a)}+O\left(b^{-2}\right) .
$$

This explains the similarity in the numerical results above. This suggests, that the Poisson distribution might be the right claim number model if the parameter value $r$ is large and $\lambda$ is small in the negative binomial distribution.

\section{REFERENCES}

Abramowitz, M. and Stegun, I. (1972) Handbook of Mathematical Functions, with Formulas, Graphs and Mathematical Tables. Dover, New York.

AMMETER, H. (1964a) Note concerning the distribution function of the total loss excluding the largest individual claims. ASTIN Bulletin 3, 132-143.

AMMETER, H. (1964b) The rating of "largest claim" reinsurance covers. Quarterly letter from the Allgemeene Reinsurance Companies, Jubilee number 9, 5-17.

BerLinger, B. (1972) Largest claims reinsurance (LCR): A quick method to calculate LCR-risk rates from excess of loss risk rates. ASTIN Bulletin 10, 54-58. 
Ciminelli, E. (1976) On the distribution of the highest claims and its application to the automobile insurance liability. Transactions of the 20th International Congress of Actuaries, 501-517.

David, H.A. (1970) Order Statistics. John Wiley \& Sons, New York.

Daykin, C.D., Pentikäinen, T. and Pesonen, M. (1994) Practical Risk Theory for Actuaries. Chapman \& Hall, London.

KREMER, E. (1982) Rating of largest claims and ECOMOR reinsurance treaties for large portfolios. ASTIN Bulletin 13, 47-56.

KREMER, E. (1984) An asymptotic formula for the net premium of some reinsurance treaties. Scandinavian Actuarial Journal 1984, 11-22.

KREMER, E. (1985) Finite formulae for the premium of the general reinsurance treaty based on ordered claims. Insurance: Mathematics and Economics 4, 233-238.

KREMER, E. (1986) Simple formulas for the premiums of the LCR and ECOMOR treaties under exponential claim sizes. Blätter der Deutschen Gesellschaft für Versicherungsmathematik 17, 457469.

KREMER, E. (1988a) Rückversicherungsprämien unter Verallgemeinerten Schadenzahlverteilungen. Transactions of the 23rd International Congress of Actuaries 73-79.

KREMER, E. (1988b) A general bound for the net premium of the largest claims reinsurance covers. ASTIN Bulletin 18, 69-78.

KuPPER, J. (1971) Contributions to the theory of the largest claim cover. ASTIN Bulletin 6, 134-146.

RytgaArd, M. (1990) Estimation in the Pareto distribution. ASTIN Bulletin 20, 201-216.

Raoul M. Berglund

Abo Akademi University

Department of Mathematics

Fänriksgatan $3 B$

20500 Åbo

Finland 